PHYSICAL REVIEW D 95, 099902(E) (2017)

\title{
Erratum: Limits on nonlinear electrodynamics [Phys. Rev. D 93, 093020 (2016)]
}

M. Fouché, R. Battesti, and C. Rizzo

(Received 10 May 2017; published 25 May 2017)

DOI: $10.1103 /$ PhysRevD.95.099902

In our paper, we made a few typing errors. On page 2, Eq. (18) should be replaced by:

$$
\mathbf{M}=\frac{\partial \mathcal{L}}{\partial \mathbf{B}}+\frac{\mathbf{B}}{\mu_{0}} .
$$

In Eq. (30) on page 4, the parameter $n^{2}$ in the $(2,1)$ matrix term should be removed and placed in the $(2,2)$ matrix term. The equation should be changed to:

$$
\left(\begin{array}{cc}
n^{2}\left(\frac{4 c_{2,0}}{\mu_{0}} B_{0}^{2}-1\right)+2+\frac{2\left(c_{0,2}-2 c_{2,0}\right)}{\mu_{0}} B_{0}^{2} & \frac{2 n c_{1,1}}{\mu_{0}} B_{0}^{2} \\
\frac{2 n c_{1,1}}{\mu_{0}} B_{0}^{2} & n^{2}\left(\frac{12 c_{2,0}}{\mu_{0}} B_{0}^{2}-1\right)+2-\frac{4 c_{2,0}}{\mu_{0}} B_{0}^{2}
\end{array}\right) \mathbf{E}_{\omega}=\mathbf{E}_{\omega} .
$$

Finally, in Eq. (82) on page 9, the $\mu_{0}$ on the last term should be removed. One obtains:

$$
\mathbf{B}_{\mathrm{V}}=\mathbf{B}\left[1-c_{2,0} \frac{Q^{2}}{4 \pi^{2} \epsilon_{0} r^{4}}\right]+c_{0,2} B(\theta=0) \frac{Q^{2} \cos \theta}{8 \pi^{2} \epsilon_{0} r^{4}} \mathbf{e}_{r} .
$$

These changes have no consequences either on the other equations or on the results.

Additionally, it appears that the excluded region due to the Lamb shift measurements and plotted in Fig. 3 on page 10 was underestimated. The constrained value $c_{2,0}<10 \times c_{2,0}^{\mathrm{HE}}$ obtained using the measurement of the $1 \mathrm{~S}-2 \mathrm{~S}$ Lamb-shift in

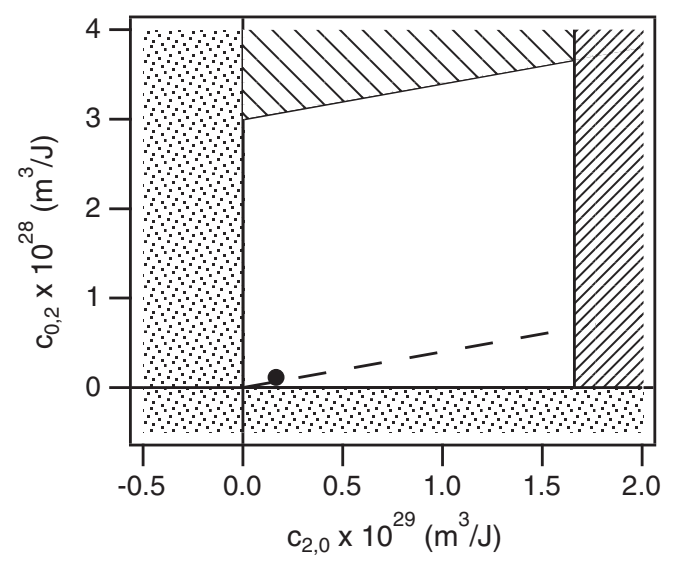

\footnotetext{
- Heisenberg-Euler prediction

- - Born-Infeld prediction

$\therefore$ Exluded region due to $\mathrm{n}_{/ /}$and $\mathrm{n}_{\perp}>1$

$\triangle \quad$ Exluded regions with Cotton-Mouton experiments
}

Exluded region with Lamb shift measurements

FIG. 1. Best experimental limits on $c_{0,2}$ and $c_{2,0}$ parameters. 
hydrogen, and established in this paper, is correct. But the plotted excluded region was calculated using $c_{0,2}^{\mathrm{HE}}$ instead of $c_{2,0}^{\mathrm{HE}}$. The corrected results are plotted in Fig. 1. However, it does not change our conclusion, in particular the fact that the Wichmann-Kroll correction has not yet been tested.

The authors thank Kazunori Shibata for identifying these errors. 